\title{
An Intrinsic Description of the Nonlinear Aeroelasticity of Very Flexible Wings
}

\author{
Rafael Palacios* \\ Imperial College, London, SW7 2AZ, United Kingdom \\ Bogdan I. Epureanu ${ }^{\dagger}$ \\ Unviersity of Michigan, Ann Arbor, Michigan 48109-2125, United States
}

\begin{abstract}
A modal solution is presented to the aeroelastic equations of very flexible wings in intrinsic form, that is written using inertial velocities and strains as primary variables. After assuming 2-D thin-airfoil aerodynamics on the wing sections, it is shown that the equations of motion can be written in canonical state-space form on the intrinsic modal coordinates without any matrix inversion and including only quadratic nonlinearities. Flutter characteristics are readily obtained from a linearized description of the dynamics equations, and the approach provides an efficient way of computing the nonlinear response with large wing displacements. Both situations are exemplified numerically on the Golang wing. The use of modal coordinates will serve to highlight some of the particular characteristics of the use of intrinsic beam solutions in aeroelastic problems with geometrical nonlinearities.
\end{abstract}

\section{Introduction}

The development of very-long-endurance UAVs with flexible high-aspect-ratio wings requires efficient aeroelastic models that include geometrically-nonlinear structural effects. They are commonly built from combining nonlinear beam models and lifting-line aerodynamics. ${ }^{1-3}$ A major complexity on the nonlinear structural dynamics solution lies in the need of parameterize and compute time-accurate finite rotations along the wing. Once a parameterization has been picked (quaternions, Euler angles, Rodrigues parameters, etc.), the resulting equations of motion are given in terms of transcendental functions, which require typically rather involved solutions process. This problem also appears in rigid-body dynamics, for which the orientation of the body needs to be computed. The traditional solution is to write the equations in terms of linear and angular velocities (the Euler-Lagrange equations of motion), leaving the rotations as secondary variables which are computed from a subsequent integration of the angular velocities. ${ }^{4}$

A similar approach is possible if the geometrically-exact beam equations are written in intrinsic form. ${ }^{5,6}$ This approach draws from Kirchhoff's analogy between the spatial and time derivatives, ${ }^{7}$ and uses a twofield description of the beam dynamics on first-derivatives, i.e., strains (internal forces) and velocities. It results in a formulation that closely resembles that of rigid-body dynamics, with first order equations of motion in both beam strains and velocities. As in rigid-body dynamics, the solution process is closed by the propagation equations to obtain displacement and rotations. This can be done by either the instantaneous spatial integration of the space derivatives (strains), ${ }^{8}$ as in Frenet-Serret formulas of differential geometry; or by time integration of the time derivatives (velocities), as in rigid-body dynamics. Finally, a compatibility equation between strains and velocities is added to ensure uniqueness of the solution. ${ }^{6}$

Therefore, for structures subject to follower forces the intrinsic equations can be used to track the dynamics without actually evaluating the local rotations, which only need to be computed at the post-processing level and do not affect the convergence rate and robustness of the solution algorithms. A recent paper by the first author ${ }^{9}$ has investigated the geometrically-nonlinear free vibrations of composite beams, showing that an intrinsic description simplifies quite significantly the evaluation of nonlinear normal modes. This modeling advantage can be retained in aeroelastic problems: Using lifting-line models, aerodynamic forces

\footnotetext{
*Lecturer, Department of Aeronautics, AIAA Member.

${ }^{\dagger}$ Associate Professor, Department of Mechanical Engineering, AIAA Member.
} 
can be written as follower forces that depend on the local instantaneous induced angle of attack of the wing airfoil. Noting that the induced angle of attack can be written as a function of the local inertia velocity in their components in a frame linked to the airfoil, one can write the aeroelastic equations of motion with structural nonlinearities purely in terms of velocities. This approach has been already followed by Patil, Hodges and co-workers ${ }^{8,10-12}$ to obtain compact flight dynamics models of very flexible aircraft. Those authors used a finite-difference approximation to the spatial component of the equations of motion, and also extended the analysis to cases with closed kinematic chains (which requires the enforcement of a unique solution along multiple load paths) using an incremental solution approach. ${ }^{12}$ In this paper, the intrinsic form of the nonlinear aeroelastic equations of motion will be projected on the linear normal modes (also expressed in intrinsic variables) of the flexible wing, which will be used to obtain a reduced-order model for nonlinear time-domain and stability analyses. Numerical examples will be finally used to validate and evaluate the performance of the solution procedure, that will be compared with results from conventional displacement-based aeroelastic models.

\section{Intrinsic Aeroelastic Model}

\section{A. Structural Model}

Following Cosserat's model, a beam will be defined as a solid determined by the rigid motion of cross sections linked to a deformable reference line. There are no assumptions on the sectional material or geometric properties, other than the condition of slenderness. The intrinsic beam equations used here were originally derived by Hodges ${ }^{6}$ and will be written for this work as ${ }^{9}$

$$
\begin{aligned}
& \mathbf{m} \dot{\mathbf{x}}_{1}-\mathbf{x}_{2}^{\prime}-\mathbf{e x}_{2}+\mathcal{L}_{1}\left(\mathbf{x}_{1}\right) \mathbf{m} \mathbf{x}_{1}+\mathcal{L}_{2}\left(\mathbf{x}_{2}\right) \mathbf{c} \mathbf{x}_{2}=\mathbf{f}_{1}, \\
& \mathbf{c} \dot{\mathbf{x}}_{2}-\mathbf{x}_{1}^{\prime}+\mathbf{e}^{T} \mathbf{x}_{1}-\mathcal{L}_{1}^{T}\left(\mathbf{x}_{1}\right) \mathbf{c} \mathbf{x}_{2}=\mathbf{0},
\end{aligned}
$$

where $(\bullet)$ and $\left(\bullet^{\prime}\right)$ denote derivatives with time, $t$, and the arc length, $x$, respectively. The state vectors $\mathbf{x}_{1}$ and $\mathbf{x}_{2}$ and the force vector $\mathbf{f}_{1}$ are given by

$$
\mathbf{x}_{1}=\left\{\begin{array}{l}
\mathbf{V} \\
\boldsymbol{\Omega}
\end{array}\right\}, \quad \mathbf{x}_{2}=\left\{\begin{array}{c}
\mathbf{F} \\
\mathbf{M}
\end{array}\right\}, \text { and } \mathbf{f}_{1}=\left\{\begin{array}{c}
f_{\boldsymbol{a}} \\
\boldsymbol{m}_{\boldsymbol{a}}
\end{array}\right\}
$$

where $\mathbf{V}(x, t)$ and $\boldsymbol{\Omega}(x, t)$ are the translational and angular inertial velocities, $\mathbf{F}(x, t)$ and $\mathbf{M}(x, t)$ are the sectional internal forces and moments, and $\boldsymbol{f}_{\boldsymbol{a}}$ and $\boldsymbol{m}_{\boldsymbol{a}}$ are the applied forces and moments per unit length, respectively. All vectors are expressed in their components in the local (deformed) material frame. The coefficients in the equations are the sectional mass $(\mathbf{m})$ and flexibility $(\mathbf{c})$ matrices, which are obtained from a cross-sectional homogenization process. ${ }^{13}$ The constant matrix $\mathbf{e}$ and the matrix operators $\mathcal{L}_{1}$ and $\mathcal{L}_{2}$ are finally defined as

$$
\mathbf{e}=\left[\begin{array}{cc}
0 & 0 \\
\tilde{\mathbf{e}}_{1} & 0
\end{array}\right], \quad \mathcal{L}_{1}\left(\mathbf{x}_{1}\right)=\left[\begin{array}{cc}
\tilde{\mathbf{\Omega}} & 0 \\
\tilde{\mathbf{V}} & \tilde{\mathbf{\Omega}}
\end{array}\right], \quad \text { and } \quad \mathcal{L}_{2}\left(\mathbf{x}_{2}\right)=\left[\begin{array}{cc}
0 & \tilde{\mathbf{F}} \\
\tilde{\mathbf{F}} & \tilde{\mathbf{M}}
\end{array}\right]
$$

where $\boldsymbol{\bullet}$ is the skew-symmetric (or cross-product) operator and $\boldsymbol{e}_{\mathbf{1}}=\{1,0,0\}^{T}$. The first equation in (1) is the actual equation of motion, while the second is a kinematic compatibility condition. They need to be solved with the problem boundary and initial conditions, which are also written in terms of velocities and forces. ${ }^{6}$ In this work, we will consider cantilever wings of length $L$, for which the natural boundary conditions are simply

$$
\begin{aligned}
\mathbf{x}_{1}(0, t) & =0, \\
\mathbf{x}_{2}(L, t) & =0 .
\end{aligned}
$$

In an intrinsic description, displacements and rotations are dependent variables, which only appear explicitly if the applied forces and moments in Eq. (1) depend on them and are obtained from time integration of the inertial velocities ${ }^{9}$ (or as spatial integration of the sectional forces and moments). This is the same post-processing done by most solution methods in rigid-body dynamics simulations. ${ }^{4}$ 


\section{B. Aerodynamic Model}

The aerodynamic loads are approximated from 2-D unsteady thin-airfoil theory. The wing has constant chord, $2 b$ and is initially straight along the $x$ axis, which goes from root to tip and cuts the airfoils at a distance $a b$ aft the aerodynamic center (located at the 1/4-chord). The airfoils are thus in the plane $y-z$, with $y$ taken along the zero-lift line and pointing towards the leading edge. This definition is also used to define the local material frame for the structural states defined in Eq. (2). The three components of the translational and angular velocity vector will be then

$$
\mathbf{V}=\left\{\begin{array}{c}
V_{x} \\
V_{y} \\
V_{z}
\end{array}\right\} \quad \text { and } \boldsymbol{\Omega}=\left\{\begin{array}{l}
\Omega_{x} \\
\Omega_{y} \\
\Omega_{z}
\end{array}\right\}
$$

If apparent mass effects are neglected, the aerodynamic forces and moments per unit length introduced in the last term of Eq. (2) (given also in their components in a frame rigidly linked to the airfoil) can be approximated by

$$
f_{\boldsymbol{a}}(x)=\rho_{\infty} b V_{y}^{2}\left\{\begin{array}{c}
0 \\
-c_{d 0}-\frac{V_{z}}{V_{y}} c_{l} \\
c_{l}
\end{array}\right\} \quad \text { and } \quad \boldsymbol{m}_{\boldsymbol{a}}(x)=\rho_{\infty} b^{2} V_{y}^{2}\left\{\begin{array}{c}
a c_{l}-\frac{\pi}{2} \frac{b \Omega_{x}}{V_{y}} \\
0 \\
0
\end{array}\right\} .
$$

In this equation, the drag coefficient $c_{d 0}$ is assumed to have a known constant value. The instantaneous circulatory lift is obtained as

$$
c_{l}=\pi\left(\alpha_{q s}+\alpha_{i}\right),
$$

where $\alpha_{q s}$ and $\alpha_{i}$ will be referred to as the effective and induced angle of attack on the airfoil, respectively. For a wing moving in still air, the effective angle of attack will be obtained as a function of the components of the inertial velocity vector at the $3 / 4$-chord point and in the plane of the airfoil, as

$$
\alpha_{q s}=\frac{b}{V_{y}} \kappa^{T} \mathbf{x}_{1},
$$

with

$$
\boldsymbol{\kappa}^{T}=\left\{\begin{array}{llllll}
0 & 0 & -\frac{1}{b} & (1-a) & 0 & 0
\end{array}\right\} .
$$

The induced angle of attack is obtained from an indicial-response approximation to the lift due to a step change of angle of attack. ${ }^{14}$ It will be written as

$$
\alpha_{i}=2 \sum_{n=1}^{N_{a}} A_{n} b_{n} \lambda_{n}
$$

where $A_{n}$ and $b_{n}$ are the coefficients of a rational-function approximation to Wagner's function. For $N_{a}=2$, it will be $b_{1}=0.0455, b_{2}=0.3, A_{1}=0.165$, and $A_{2}=0.335$. The terms $\lambda_{n}$ are the aerodynamic lags at the local airfoil, whose evolution in time will be approximated by the differential equations,

$$
\dot{\lambda_{n}}+\omega_{n}^{a} \lambda_{n}=\boldsymbol{\kappa}^{T} \mathbf{x}_{1},
$$

for $n=1, \ldots, N_{a}$ and with $\omega_{n}^{a}=\frac{b_{n} V_{\infty}}{b}$. This assumes that the time-dependent contributions in $V_{y}$ are small compared to the free-stream velocity $V_{\infty}$. If the rate of change of the aerodynamic lags is very small compared to the aerodynamic time scale (i.e., $\frac{1}{\omega_{n}^{a}}$ ), then it can be further assumed that $\dot{\lambda}_{n}=0$, quasi-steady aerodynamics. Using the fact that $\sum A_{n}=\frac{1}{2}$, one obtains from Eqs. (10) and (11) that, under quasi-steady aerodynamic assumptions, it is $\alpha_{i}=\alpha_{q s}$. For the general unsteady case, the aerodynamic forces can be finally written in compact form as

$$
\boldsymbol{f}_{\mathbf{1}}=\pi \rho_{\infty} b\left[\mathcal{A}_{1}\left(\mathbf{x}_{1}\right)+\alpha_{i} \mathcal{A}_{2}\left(\mathbf{x}_{1}\right)\right] \mathbf{x}_{1},
$$


with

$$
\begin{aligned}
& \mathcal{A}_{1}\left(\mathbf{x}_{1}\right)=\left[\begin{array}{cccccc}
0 & 0 & 0 & 0 & 0 & 0 \\
0 & -\frac{c_{d 0}}{\pi} V_{y} & V_{z} & -b(1-a) V_{z} & 0 & 0 \\
0 & -V_{z} & 0 & b(1-a) V_{y} & 0 & 0 \\
0 & -a b V_{z} & 0 & \left(a-a^{2}-\frac{1}{2}\right) b^{2} V_{y} & 0 & 0 \\
0 & 0 & 0 & 0 & 0 & 0 \\
0 & 0 & 0 & 0 & 0 & 0
\end{array}\right], \\
& \mathcal{A}_{2}\left(\mathbf{x}_{1}\right)=\left[\begin{array}{cccccc}
0 & 0 & 0 & 0 & 0 & 0 \\
0 & 0 & -V_{y} & 0 & 0 & 0 \\
0 & V_{y} & 0 & 0 & 0 & 0 \\
0 & a b V_{y} & 0 & 0 & 0 & 0 \\
0 & 0 & 0 & 0 & 0 & 0 \\
0 & 0 & 0 & 0 & 0 & 0
\end{array}\right]
\end{aligned}
$$

The force vector in the nonlinear aeroelastic system defined by Eqs. (1) is then obtained from Eq. (12) with the induced angle of attack given in (10). Similar expressions were obtained by Shang and Hodges ${ }^{15}$ and Patil and Hodges ${ }^{10}$ using an approximation to the inflow velocities using Glauert expansions. ${ }^{16}$ Some small angle approximations were introduced here which were deemed consistent with the overall level of approximation of this low-fidelity approach. As it can be seen, under the assumption of 2-D aerodynamics, the force vector given by Eq. (12) only depends on the sectional inertial velocities projected on the (local) material reference frame. Phase shifts in the unsteady aerodynamics are introduced by the aerodynamic lags given by Eq. (11), which are also a function of the time history of the local inertial velocities.

\section{Nonlinear Equations in Intrinsic Modal Coordinates}

The aeroelastic equations of motion for the very flexible wing are then described by Eq. (1), with the boundary conditions (4) and forcing terms given by Eqs. (10)-(12). These nonlinear equations will be solved after projection on the vibration modes of the linear structure, which are obtained next.

\section{A. Linear Normal Modes}

A detailed description of a methodology for evaluation of the beam Linear Normal Modes (LNMs) from the intrinsic equations has been presented in a previous work by the first author. ${ }^{9}$ They are obtained from the unforced equations of motion linearized around $\mathbf{x}=0$. In that case Eq. (1) is written as

$$
\begin{aligned}
\mathbf{m} \dot{\mathbf{x}}_{1}-\mathbf{x}_{2}^{\prime}-\mathbf{e x}_{2}=\mathbf{0} \\
\mathbf{c} \dot{\mathbf{x}}_{2}-\mathbf{x}_{1}^{\prime}+\mathbf{e}^{T} \mathbf{x}_{1}=\mathbf{0}
\end{aligned}
$$

The LNMs of the problem are the nontrivial solutions to this homogeneous equation that satisfy the spatial boundary conditions. For the particular case of a uniform beam with constant properties $\mathbf{m}$ and $\mathbf{c}$ are constant matrices and the solution can be obtained as ${ }^{9}$

$$
\left\{\begin{array}{l}
\boldsymbol{\Phi}_{1 j} \\
\boldsymbol{\Phi}_{2 j}
\end{array}\right\}=e^{\mathbf{A}\left(\omega_{j}\right) x}\left\{\begin{array}{l}
\boldsymbol{\Phi}_{1 j}(0) \\
\boldsymbol{\Phi}_{2 j}(0)
\end{array}\right\},
$$

with

$$
\mathbf{A}\left(\omega_{j}\right)=\left[\begin{array}{cc}
\mathbf{e}^{T} & -\omega_{j} \mathbf{c} \\
\omega_{j} \mathbf{m} & -\mathbf{e}
\end{array}\right] .
$$

The values of $\boldsymbol{\Phi}_{j}(0)$ and $\omega_{j}$ are obtained from imposing the specific boundary conditions of the problem and they uniquely determine the $j^{\text {th }}$ LNM. For instance, for a cantilever beam of length $L$, the are obtained 
from the nontrivial solutions of

$$
\left[\begin{array}{cc|cc}
e^{\mathbf{A}\left(\omega_{j}\right) L} & -\mathbf{I} & \mathbf{0} \\
& \mathbf{0} & -\mathbf{I} \\
\hline \mathbf{I} & \mathbf{0} & \mathbf{0} & \mathbf{0} \\
\mathbf{0} & \mathbf{0} & \mathbf{0} & \mathbf{I}
\end{array}\right]\left\{\begin{array}{l}
\boldsymbol{\Phi}_{1 j}(0) \\
\boldsymbol{\Phi}_{2 j}(0) \\
\boldsymbol{\Phi}_{1 j}(L) \\
\boldsymbol{\Phi}_{2 j}(L)
\end{array}\right\}=\left\{\begin{array}{l}
\mathbf{0} \\
\mathbf{0} \\
\mathbf{0} \\
\mathbf{0}
\end{array}\right\}
$$

Once those coefficients (13 per mode) are obtained, Eq. (15) provides an analytical expression for the mode shapes for straight beams of constant section. This is done without any assumption on their sectional properties, that is, $\mathbf{m}$ and $\mathbf{c}$ can be full matrices. Moreover, both matrices will be symmetric and the exponential matrix in Eq. (15) is easily evaluated using similarity transformations. A consistent normalization of the modes, which will be used here, is ${ }^{9}$

$$
\begin{gathered}
\int_{0}^{L} \boldsymbol{\Phi}_{1 j}^{T} \mathbf{m} \boldsymbol{\Phi}_{1 k} d x=\delta_{j k}, \\
\int_{0}^{L} \boldsymbol{\Phi}_{2 j}^{T} \mathbf{c} \boldsymbol{\Phi}_{2 k} d x=\delta_{j k} .
\end{gathered}
$$

In textbook cases (e.g. isotropic Euler-Bernoulli beams), Eq. (15) reduces to the derivatives (in time and space) of well-known expressions in the beam displacements and rotations. As an example, the Appendix includes the normalized analytical expressions for the intrinsic LNMs of a beam clamped on a base which is free to translate in one direction. That particular solution will be later used in this work. Once the LNMs are obtained (and normalized with the mass and compliance matrices ${ }^{9}$ ), the state vectors defined in Eq. (2) can be finally projected on this modal basis as

$$
\begin{aligned}
& \mathbf{x}_{1}(x, t)=\sum_{j=0}^{\infty} \boldsymbol{\Phi}_{1 j}(x) q_{1 j}(t), \\
& \mathbf{x}_{2}(x, t)=\sum_{j=0}^{\infty} \boldsymbol{\Phi}_{2 j}(x) q_{2 j}(t),
\end{aligned}
$$

where $\left(q_{1 j}, q_{2 j}\right)$ are pairs of intrinsic modal coordinates. Since this is a first-order theory, each natural frequency will be associated to two generalized coordinates.

\section{B. Nonlinear Aeroelastic Equations in the Modal Basis}

The modal expansion given by Eq. (19) will be introduced now on the structural degrees of freedom in Eqs. (1). According to Eq. (15), the aerodynamic lags at a given section will be written as

$$
\lambda_{n}=\sum_{j=0}^{\infty} \boldsymbol{\kappa}^{T} \boldsymbol{\Phi}_{1 j}(x) q_{n j}^{a}(t),
$$

After imposing orthogonality conditions on the mode shapes, ${ }^{9}$ the aeroelastic equations of motion (1) can be written in intrinsic modal coordinates, as

$$
\begin{aligned}
& \dot{q}_{1 j}-\omega_{j} q_{2 j}+\sum_{k=0}^{\infty} \sum_{l=0}^{\infty}\left(\beta_{1}^{j k l}-\rho_{\infty} \mu_{1}^{j k l}\right) q_{1 k} q_{1 l}+\sum_{k=0}^{\infty} \sum_{l=0}^{\infty} \beta_{2}^{j k l} q_{2 k} q_{2 l}+ \\
&-\sum_{n=1}^{N_{a}}\left(2 A_{n} b_{n} \sum_{k=0}^{\infty} \sum_{l=0}^{\infty} \rho_{\infty} \mu_{2}^{j k l} q_{1 k} q_{n l}^{a}\right)=Q_{1 j}, \\
& \dot{q}_{2 j}+\omega_{j} q_{1 j}- \sum_{k=0}^{\infty} \sum_{l=0}^{\infty} \beta_{2}^{k j l} q_{1 k} q_{2 l}=0, \\
& \dot{q}_{n j}^{a}+\omega_{n j}^{a} q_{n j}^{a}-q_{1 j}=0,
\end{aligned}
$$


for $j=0,1,2, \ldots$ and $n=1, \ldots, N_{a}$, and with

$$
\begin{aligned}
& \beta_{1}^{j k l}=\int_{0}^{L} \boldsymbol{\Phi}_{1 j}^{T} \mathcal{L}_{1}\left(\boldsymbol{\Phi}_{1 k}\right) \mathbf{m} \boldsymbol{\Phi}_{1 l} d x, \\
& \beta_{2}^{j k l}=\int_{0}^{L} \boldsymbol{\Phi}_{1 j}^{T} \mathcal{L}_{2}\left(\boldsymbol{\Phi}_{2 k}\right) \mathbf{c} \boldsymbol{\Phi}_{2 l} d x, \\
& \mu_{1}^{j k l}=\int_{0}^{L} \boldsymbol{\Phi}_{1 j}^{T} \mathcal{A}_{1}\left(\boldsymbol{\Phi}_{1 k}\right) \pi b \boldsymbol{\Phi}_{1 l} d x, \\
& \mu_{2}^{j k l}=\int_{0}^{L}\left(\boldsymbol{\Phi}_{1 j}^{T} \mathcal{A}_{2}\left(\mathbf{x}_{1}(x, 0)\right) \pi b \boldsymbol{\Phi}_{1 k}\right)\left(\boldsymbol{\kappa}^{T} \boldsymbol{\Phi}_{1 l}\right) d x .
\end{aligned}
$$

The last equation was built on the assumption that the horizontal velocity can be approximated by the initial velocity (the free-stream velocity) in the evaluation of $\mathcal{A}_{2}$ in Eq. (13). This is consistent with the approximation done in the differential equation for the aerodynamic lags, Eq. (11). The generalized forces on the first equation in (21) are computed as $Q_{1 j}=\int_{0}^{L} \boldsymbol{\Phi}_{1 j}^{T} \mathbf{f}_{1} d x$. After truncation of the expansions (19) to $N_{s}$ modes, the system dynamics are described by $2 N_{s}$ structural states and $N_{a} \times N_{s}$ aerodynamic states. Note that for constant properties along the wing span, the mode shapes needed to evaluate these integrals, are known analytically from Eq. (15). Finally, if quasi-steady aerodynamic assumptions can be made, it will be $\dot{q}_{n j}^{2}=0$ in the last equation of Eq. (21) and the aerodynamic states are obtained as $q_{n j}^{a}=\frac{q_{1 j}}{\omega_{n}^{a}}$.

\section{Kinematic Constraints}

The intrinsic model describes the wing dynamics using the local inertial velocities in the material frame. In many cases, the problem needs to be solved with kinematic constraints, such as in the case of wings with constant forward-flight velocity (or mounted in a wind tunnel). Those constraints can be imposed in at least two ways: 1) by modifying the boundary conditions of Eq. (1) at $x=0$; or 2) by introducing Lagrange multipliers on the modal Eqs. (21) obtained using free-end boundary conditions. In this last case, the first of the equations (21) is rewritten as

$$
\dot{q}_{1 j}=\mathcal{F}_{1 j}\left(\mathbf{q}_{1}, \mathbf{q}_{2}, \mathbf{q}^{a} ; \rho_{\infty}\right)+Q_{1 j}+\boldsymbol{\Lambda}^{T} \boldsymbol{\Phi}_{1 j}(0),
$$

where $\boldsymbol{\Lambda}$ is the vector of Lagrange multipliers introduced to enforce the kinematic constraints at the wing root, which are imposed as $\mathbf{x}_{1}(0, t)=\mathbf{x}_{10}(t)$. In modal coordinates, that is

$$
\sum_{k=0}^{\infty} \boldsymbol{\Phi}_{1 k}(0) q_{1 k}-\mathbf{x}_{10}(t)=0
$$

The problem is the well posed by solving together Eqs. (23) and (24). This defines in set of differentialalgebraic equations, that it can be easily transformed into a differential form by taking derivatives in the last equation and substituting $\dot{q}_{1 j}$ from Eq. (23) into the resulting expression. This gives an expression to evaluate the Lagrange multipliers, as

$$
\sum_{k=0}^{\infty}\left(\boldsymbol{\Lambda}^{T} \boldsymbol{\Phi}_{1 k}(0)\right) \boldsymbol{\Phi}_{1 k}(0)=\dot{\mathbf{x}}_{10}(t)-\sum_{k=0}^{\infty}\left(\mathcal{F}_{1 k}+Q_{1 k}\right) \boldsymbol{\Phi}_{1 k}(0) .
$$

Eq. (25) defines a linear system of equation that from which the Lagrange multipliers can be directly solved. The constraints can then be finally enforced on Eq. (23) without increasing the number of states of the problem.

\section{Numerical Studies}

As mentioned in the introduction, the intrinsic equations are particularly advantageous for problems with open kinematic chains and without dead loads. This section will investigate two such scenarios: forced vibrations induced by the beam support, and aeroelastic stability of cantilever wings. The objective is first to assess the validity of the approach, by comparing its results with published ones, and second to explore the specific characteristics of the intrinsic formulation in modal coordinates. 


\section{A. Forced vibrations in vacuum of a cantilever beam on a moving base}

The configuration experimentally studied by $\mathrm{Pai}^{17}$ will be first considered. It is a prismatic cantilever beam made of Titanium (i.e., mass density $4430 \mathrm{~kg} / \mathrm{m}^{3}$, Young modulus $127 \mathrm{GPa}$, Poisson ratio 0.36) with dimensions $479.0 \times 0.45 \times 50.8 \mathrm{~mm}$ (along axis $x, y$, and $z$, respectively). This beam is clamped onto a moving base with a prescribed translational velocity along the $y$ axis. This test case will therefore serve to exercise the nonlinear structural equations in intrinsic modal coordinates with constraints for the moving support. The boundary conditions to the problem will therefore be

$$
\begin{aligned}
\mathbf{x}_{1}(0) & =\mathbf{x}_{10}, \\
\mathbf{x}_{2}(L) & =0 .
\end{aligned}
$$

In particular, for this problem it will be $\mathbf{x}_{10}^{T}=\left\{0, V_{0} \sin \left(\Omega_{0} t\right), 0,0,0,0\right\}$. It is a plane problem and the beam kinematics can be captured by a combination of axial modes with clamped-free boundary conditions and in-plane bending modes with slide-free boundary conditions. Closed-form analytical expressions for these mode shapes exist and can be found in the Appendix. Finally, condition (26) will be applied as a kinematic constraint as described in section III.C. Figure 1 shows the instantaneous velocities along the beam on 50 consecutive steps during one period of base oscillation for $\Omega_{0}=9 \mathrm{~Hz}, V_{0}=0.1399 \mathrm{~m} / \mathrm{s}$ and $\rho_{\infty}=0$. It compares three sets of results: 1) the present solution in intrinsic modal coordinates using 5 axial and 5 in-plane bending modes; 2) results of a finite-element solution based on displacements and the Cartesian rotation vector as independent variables ${ }^{18}$ (using 25 spanwise elements); 3 ) the laser-vibrometer experimental results of Pai. ${ }^{17}$ A total of 100 time steps per cycle of excitation are taken in the numerical solutions. Figure 1 shows the local in-plane components of the translational velocity in the instantaneous material frame and the spanwise distribution of angular velocity. Note that the nonlinear effects are small and were not recorded in the experimental setup. They have been included for both numerical solutions. Excellent agreement is obtained between all three results.
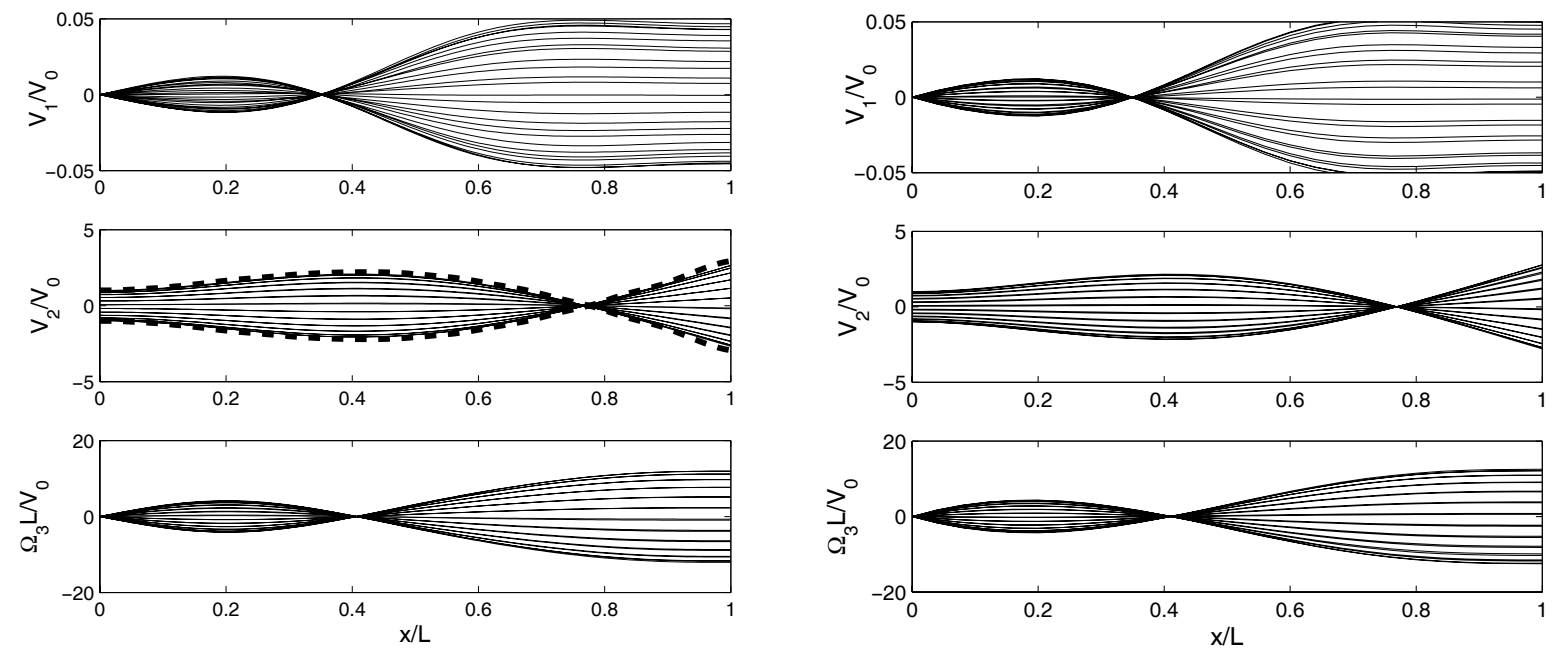

(a) Intrinsic Modal Solution (dotted line for available ${ }^{17}$ experimental data)

(b) Finite-Element Solution

Figure 1: Velocity profiles (components in material frame) at 50 consecutive steps during one period of oscillation $\left[\Omega_{0}=9 \mathrm{~Hz}, V_{0}=0.1399 \mathrm{~m} / \mathrm{s}\right]$.

A fundamental characteristic of the present approach is that the primary variables in the problem are the velocities and internal forces/moments, expressed in their components in a body-attached reference frame. As it was also discussed above, this suffices to compute the 2-D unsteady aerodynamics of the wing airfoils. This means, in particular, that the effect of the local geometric angle of attack is not captured through the torsional vibration modes, but rather appears as the induced angle of attack created by out-of-plane bending modes. To exemplify this, the previous configuration is now subject to a constant velocity of the moving 
base $\left(V_{0}=10 \mathrm{~m} / \mathrm{s}, \Omega_{0}=0\right)$ and to distributed applied moments. To simplify the solution, the moments are chosen such that the generalized forces $\mathbf{Q}_{1}$ in Eq. (21) are zero for all modes except for the first torsional one. They are applied as harmonic functions of frequency $0.25 \mathrm{~Hz}$ and unit amplitude of the generalized force. The input was linearly ramped through two cycles of excitations to remove transient effects and the solutions were obtained with 100 time steps per cycle. Results are shown in Figure 2.
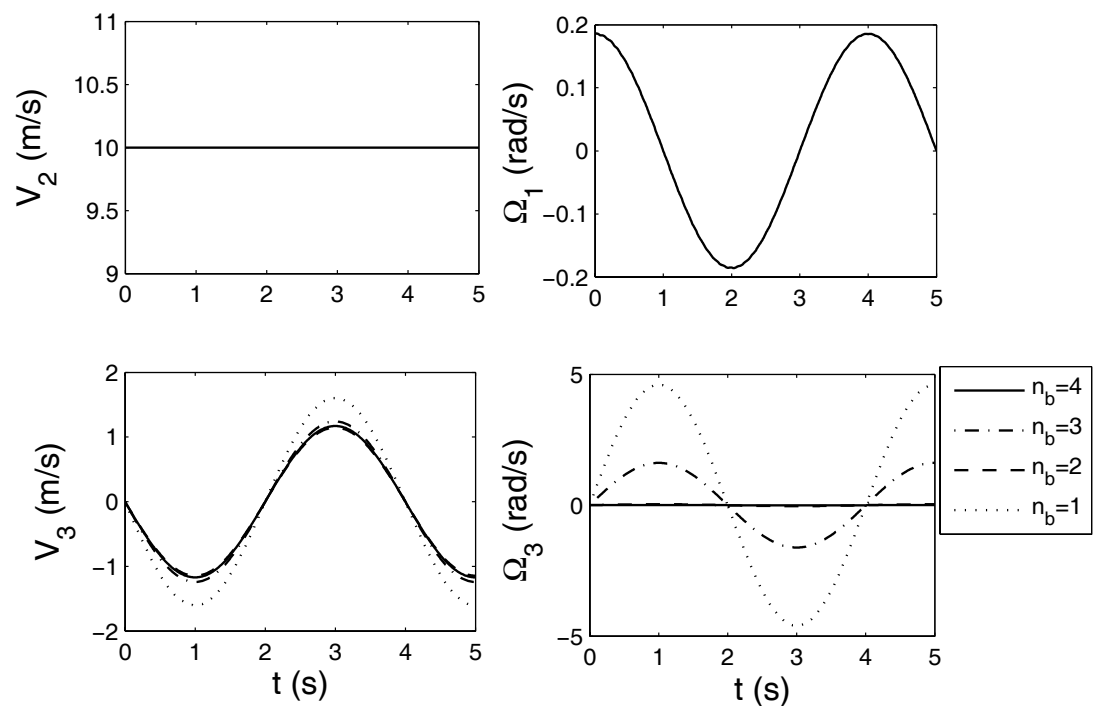

Figure 2: Non-zero tip velocities for the cantilever beam on a moving base with constant velocity $\left(V_{0}=\right.$ $10 \mathrm{~m} / \mathrm{s})$, under applied distributed moments on the first torsion mode $\left(Q_{1}=\sin \frac{\pi t}{2}\right)$, and for an increasing number, $n_{b}$, of out-of-plane bending modes.

Figure 2 compares the non-zero velocities at the beam tip $(x=L)$ obtained from the nonlinear equations (21) in vacuum. The solution includes only one torsional and one in-plane bending mode and an increasing number $\left(n_{b}\right)$ of out-of-plane bending modes. The excitation is a constant velocity (captured by the in-plane bending mode, which is a rigid body mode) and the distributed twist moment described above. The nonlinear terms in the equation evaluate the induced out-of-plane velocity on the beam due to the local geometric angle of attack. As the distribution of angles of attack in this example has the shape of the first torsional mode but it is captured by the out-of-plane bending modes, one needs several bending modes to reproduce that shape. In particular, four bending modes were needed in Figure 2 to obtain an accurate distribution of out-of-plane velocities on the beam. Integrating the angular velocity in time, one obtains the instantaneous geometric twist and the free end. The maximum value is $\theta_{1, \max }=6.8 \mathrm{deg}$, which corresponds to the maximum value of the out-of-plane velocity $V_{0} \sin \left(\theta_{1, \max }\right)=1.18 \mathrm{~m} / \mathrm{s}$ in Figure 2 .

Note finally in Figure 2 that for a (small) even number of nodes, a large bending angular velocity $\left(\Omega_{3}\right)$ is obtained. This component is due exclusively to the numerical approximation and it converges relatively slowly to zero when increasing the number of modes. These results indicate that the wing aeroelasticity in intrinsic modal coordinates will show different modal interactions than those seen in more conventional displacement-based descriptions. Particular care, through convergence studies, must be taken to ensure that the selected modal basis provides an appropriate approximation for the wing kinematics.

\section{B. Aeroelastic response of a cantilever wing}

The Goland wing ${ }^{19}$ is a numerical test case that has been extensively used as a benchmark for aeroelastic solutions with structural dynamic models based on beam elements. ${ }^{2,11,20-22}$ Its relevant properties are included in Table 1. It should be noted that this definition corresponds to the original description due to Goland, ${ }^{19}$ instead of the heavy version (often referred to as Goland ${ }^{+}$) of Eastep and Olsen. ${ }^{23}$ To compute the aeroelastic equations of motion in intrinsic coordinates, Eq. (21), motions in the plane of the wing are expressed in terms of slide-free in-plane bending LNMs. The free-stream velocity can then be introduced 
through kinematic constraints on those modes at the root. Due to the offset between the elastic axis and the cross-sectional center of gravity, the out-of-plane bending and the torsional degrees of freedom are coupled. The non-zero components first four modes bending/torsion modes are given in Figure 3. Both the velocity and force components of the modes, which have been normalized as in Eq. (18), have been included in the figure. Note that all mode shapes have both out-of-plane bending and torsional components and they match a the results obtained with different formulations. ${ }^{21}$
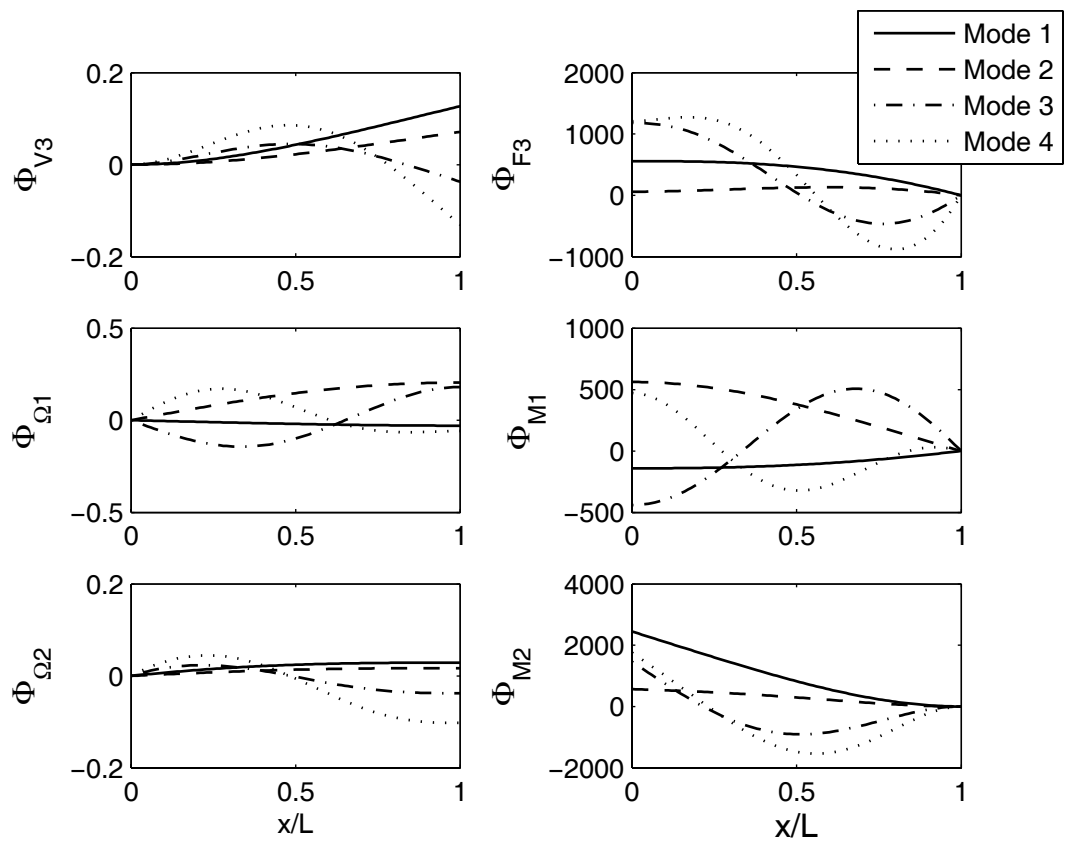

Figure 3: Non-zero components of the first four twist/out-of-plane bending modes of the Goland wing $\left[\omega_{1}=7.66 \mathrm{~Hz}, \omega_{2}=15.24 \mathrm{~Hz}, \omega_{3}=38.80 \mathrm{~Hz}, \omega_{4}=55.33 \mathrm{~Hz}\right]$.

The dynamic stability characteristics are obtained with a linearized model around the solution for constant forward flight velocity, that is, with a constant value on the first in-plane mode (it will be identified as mode 0 ). From Eq. (36) in the appendix, it is $q_{10}=V_{\infty} \sqrt{\rho A L}$ and $q_{20}=0$. The linear dynamic model will only need to include the first torsion/out-of-plane bending modes (modes 1 to $n_{b}$ ). The resulting linear equations of motion are

$$
\begin{aligned}
& \dot{q}_{1 j}=\omega_{j} q_{2 j}-q_{10} \sum_{k=1}^{n_{b}}\left[\beta_{1}^{j k 0}+\beta_{1}^{j 0 k}-\rho_{\infty}\left(\mu_{1}^{j k 0}+\mu_{1}^{j 0 k}\right)\right] q_{1 k}+q_{10} \sum_{k=1}^{n_{b}} \sum_{n=1}^{N_{a}} 2 \rho_{\infty} A_{n} b_{n}\left[\mu_{2}^{j 0 k} q_{n k}^{a}+\frac{\mu_{2}^{j k 0}}{\omega_{n}^{a}} q_{1 k}\right], \\
& \dot{q}_{2 j}=-\omega_{j} q_{1 j}+q_{10} \sum_{k=1}^{n_{b}} \beta_{2}^{0 j k} q_{2 k}, \\
& \dot{q}_{n j}^{a}=-\omega_{n}^{a} q_{n j}^{a}+q_{1 j},
\end{aligned}
$$

for $j=1, \ldots, n_{b}$. For the parameters of Goland's test case, it is $\mu_{2}^{j k 0}=0$, which further simplifies the description. For a given air density, the eigenvalues of this linear system determine the dynamic stability of the wing as a function of $q_{10}=V_{\infty} \sqrt{\rho A L}$. As it was discussed in the test case in section IV.A, several bending modes may be needed to capture the changes of local angle of attack due to the twisting of the wing. Table 2 includes the results for increasing number of modes and air density $\rho_{\infty}=1.02 \mathrm{~kg} / \mathrm{m}^{3}$ as in Ref. 19. Figure 4 shows the eigenvalues of Eq. (27) obtained with eight modes $\left(n_{b}=8\right)$ for increasing values of the free-stream velocity. In particular, the velocity part $\left(q_{1 j}\right)$ of the eigenvector of the unstable root at $V_{\infty}=141 \mathrm{~m} / \mathrm{s}$ with $n_{b}=8$ has amplitudes $(1.00,0.23,0.22,0.20,0.09,0.00,0.01,0.04)$ on the different linear modes. From this and from Table 2, it can be seen that one needs a minimum of between

\footnotetext{
${ }^{a}$ As already mentioned, the mode shapes in intrinsic variables are the time and spatial derivatives of those obtained in a formulation in displacements. The natural frequencies are the same.
} 
six to eight modes to obtain a good estimation of the flutter onset point with the intrinsic description. As a comparison, four modes have been found to be enough to estimate the flutter speed of the Goland wing with displacement-based theories. ${ }^{21}$ As a general rule, the intrinsic formulation trades a larger size of the linear problem by a simpler description of the nonlinear dynamics.

Table 1: Relevant properties of the Goland wing

\begin{tabular}{lrlr}
\hline \hline Chord, $2 b$ & $1.8288 \mathrm{~m}$ & Mass per unit length, $\rho A$ & $35.71 \mathrm{~kg} / \mathrm{m}$ \\
Semi-span, $L$ & $6.096 \mathrm{~m}$ & Moment of inertia around e.a., $\rho I_{1}$ & $8.64 \mathrm{~kg} \cdot \mathrm{m}$ \\
Elastic axis (from l.e.) & $33 \%$ chord & Torsional stiffness, $G J$ & $0.99 \times 10^{6} \mathrm{~N} \cdot \mathrm{m}^{2}$ \\
Center of gravity (from l.e.) & $43 \%$ chord & Bending stiffness, $E I_{2}$ & $9.77 \times 10^{6} \mathrm{~N} \cdot \mathrm{m}^{2}$ \\
\hline \hline
\end{tabular}

Table 2: Flutter velocity and frequency for the Goland wing $\left[\rho_{\infty}=1.02 \mathrm{~kg} / \mathrm{m}^{3}\right]$

\begin{tabular}{|c|c|c|c|}
\hline Author & Model & $V_{f}, \mathrm{~m} / \mathrm{s}$ & $\omega_{f}, \mathrm{rad} / \mathrm{s}$ \\
\hline Present $\left(n_{b}=4\right)$ & Intrinsic beam +2 -D aero (modal) & 130 & 69.1 \\
\hline Present $\left(n_{b}=6\right)$ & Intrinsic beam +2 -D aero (modal) & 140 & 69.9 \\
\hline Present $\left(n_{b}=8\right)$ & Intrinsic beam $+2-\mathrm{D}$ aero (modal) & 141 & 69.8 \\
\hline Sotoudeh et al. ${ }^{11}$ & Intrinsic beam $+2-\mathrm{D}$ aero (finite differences) & 137 & 70.1 \\
\hline Wang et $\mathrm{al}^{20}$ & Intrinsic beam + UVLM & 164 & - \\
\hline Murua et al. ${ }^{21}$ & Displacement beam + UVLM & 165 & 69 \\
\hline
\end{tabular}

Flutter results in Table 2 are compared to those of Sotoudeh et al., ${ }^{11}$ who have also used an implicit beam model together with the 2-D unsteady aerodynamic model of Peters. ${ }^{16}$ The solution of these authors is based on a finite-difference approximation to Eqs. (1), while the present one uses linear normal modes. As it can be seen both solutions compare very well. Table 2 also includes flutter results obtained using full 3-D aerodynamics coupled with beam models, in particular, using the unsteady vortex lattice method (UVLM). It is clear that the assumption of 2-D aerodynamics introduces a significant error for such a short wing span, yet this test case still serves to verify the current solution approach.

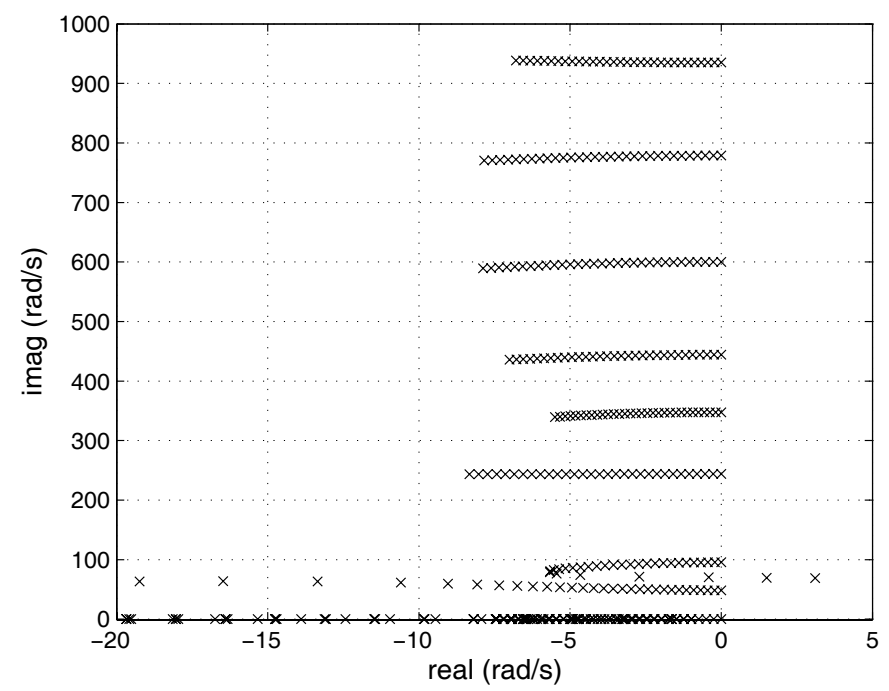

Figure 4: Root locus of the linear Goland wing with $n_{b}=8$ and $N_{a}=2$. Air speed from zero to $V_{\infty}=150$ $\mathrm{m} / \mathrm{s}$ in increments of $5 \mathrm{~m} / \mathrm{s}$. Instability occurs at $141 \mathrm{~m} / \mathrm{s}$. 
It should be finally noted that the linearized aeroelastic equations, Eqs. (27), were obtained around the forward flight conditions. This is not because of the trimming of the aircraft in steady flight (although this could be also the case) but because the modal projection was done in Eq. (19) using the modes about the zero reference state $\mathbf{x}=0$, and those include the beam inertial velocities. This is a direct consequence of the fact that the air speed is not included explicitly in the intrinsic equations. To exemplify this, the full solution to the linearized equations (about $\mathbf{x}=0$ ) and the nonlinear equations (21) is obtained in time domain for zero external forces and non-zero initial velocities on the second bending mode. These results would be relevant in the design of gust alleviation mechanisms for very flexible wings, which is one of the main potential applications of this model and may required a linearized description of the dynamics. For the nonlinear response, an axial stiffness $E A=10^{9} \mathrm{~N}$ has been added to the original wing parameters defined by Goland. ${ }^{19}$ The numerical results are obtained using an expansion with 13 linear normal modes: 4 axial, 8 out-of-plane bending/torsion modes, and the rigid-body in-plane bending mode. Note however that the absence of flexible in-plane bending modes will prevent the analysis to capture the "cosine" effect in the rotation of the blades, which was not considered important here. The solution is obtained with a fourth-order explicit Runge-Kutta solver with time step $\Delta t=\frac{0.01}{2 \pi \omega_{2}}$.
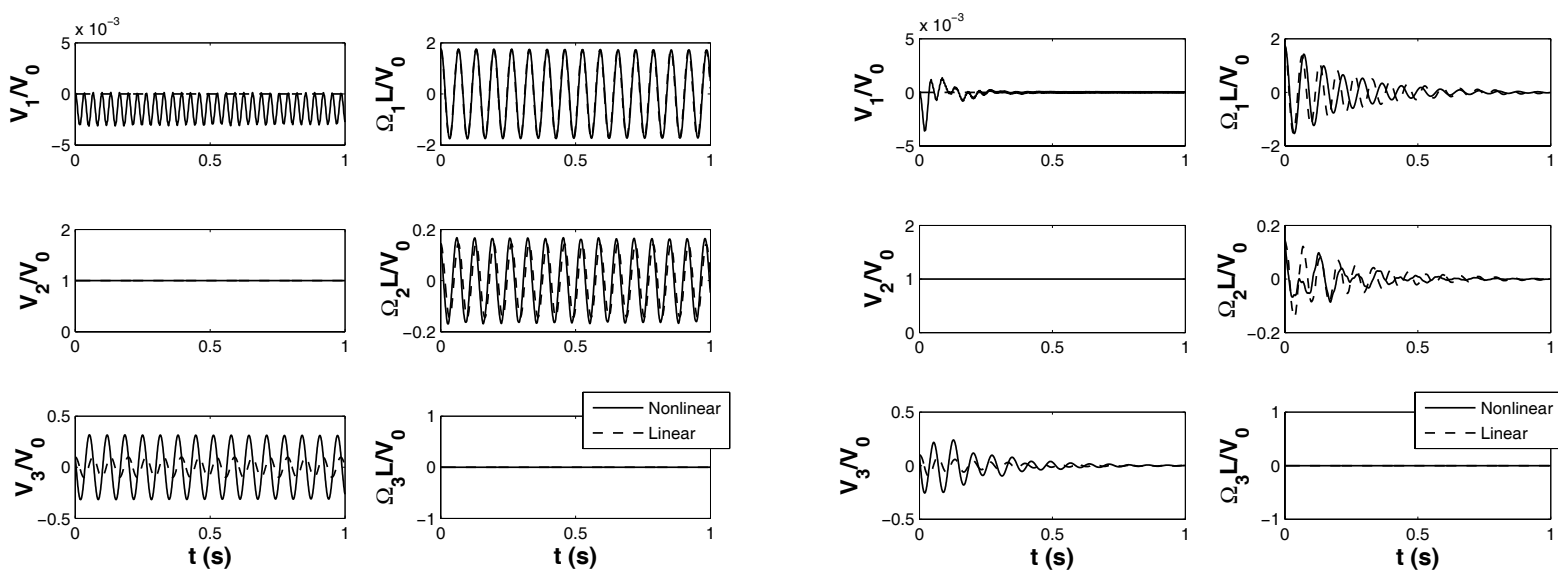

(a) $\rho_{\infty}=0$
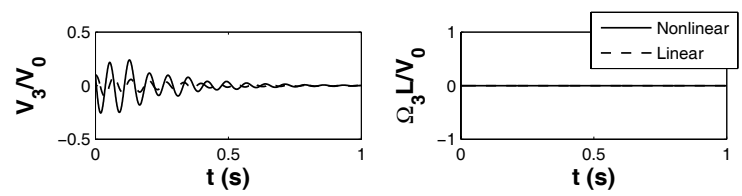

(b) $\rho_{\infty}=1.02 \mathrm{~kg} / \mathrm{m}^{3}$

Figure 5: Effect of linearization around $\mathbf{x}=0$ on the transient response of the Goland wing $\left[V_{0}=100 \mathrm{~m} / \mathrm{s}\right.$ and initial conditions on second bending mode with initial tip vertical velocity $10 \mathrm{~m} / \mathrm{s}$.

Figure 5 a shows the free vibration in vacuum for $V_{\infty}=100 \mathrm{~m} / \mathrm{s}$ and initial tip velocity of $10 \%$ of this value (this determines the amplitude of the initial conditions on the second bending mode). From the comparison between the linear and the nonlinear response, it is clear that the linearization of the modal equations about $\mathbf{x}=\mathbf{0}$ (i.e., static undeformed conditions) misses the contribution of the forward-flight velocity into the local out-of-plane axis as the wing twists. The presence of air is illustrated in Figure 5b, which corresponds to the same conditions but with $\rho_{\infty}=1.02 \mathrm{~kg} / \mathrm{m}^{3}$. As it can be seen, the aerodynamic damping has a very similar effect on both the linear and the nonlinear models, despite the larger initial oscillations in the nonlinear case. This could mean that a controller for gust alleviation designed on the linearized dynamics may perform well on different equilibrium points.

\section{Conclusions}

This work has presented a modal solution of the dynamic aeroelasticity of wings in intrinsic variables. The appeal of this approach is in its ability to deal with geometrically-nonlinear effects using a rather simple description: The equations of motion in the modal coordinates have only quadratic nonlinearities and their constant coefficients are obtained from integral expressions involving only the sectional (structural and aerodynamic) properties and the mode shapes. The assumptions are those commonly used in the literature: thin strip unsteady aerodynamics and geometrically-nonlinear beam structural models. The 
modal formulation has been presented for a straight wing with constant properties, including anisotropic elastic behavior, but it can be easily expanded to more complex geometries (e.g. a full aircraft). In that case, the analytical equations to obtain the mode shapes would need to be replaced by numerical solutions. Note however that the kinematic relations between forces/moments and velocities, on one side, and displacements and linear rotations, on the other, ensure that the linear normal modes in intrinsic coordinates will be the time and spatial (along the reference line) derivatives of the mode shapes in a displacement formulation with beam elements. It should be therefore possible to obtain the mode shapes for the intrinsic formulation directly from the results of standard finite-element packages. This should be evident from the closed-form expressions including in the appendix to this paper, but still needs to be investigated for more complex geometries.

In intrinsic variables, the only necessary information for each wing airfoil is its inertial velocity (linear and angular) and the resulting of the forces and moments created on it by the local external actions (2-D aero loads) and the rest of the structure. Although it can still be computed, there is no need to know its actual position and orientation in space. This characteristic has been retained in the projection of the equation of motion into modal coordinates, which has simplified the solution. Direct application of this procedure however, would not allow for kinematic constraints on the wing, which could be as simple as a constant translational velocity (i.e., forward flight case). To overcome this, the paper has introduced a simple mechanism to enforce root motions, based on free-free modes and Lagrange multipliers, that keeps the solution structure of the problem. Numerical results have shown the validity of the approach.

A final aspect that must be remarked is that the solution to the intrinsic equations may not be always intuitive for the aeroelastician. First, angle of attack effects are split between torsional (angular rate effect) and bending (induced velocity effect) degrees of freedom. This also alters the description of the modal interactions occurring in linear stability (flutter) analysis. Second, by solving the equations of motion in the local material frame, any forcing that depends on an external reference (from the forward flight velocity, discussed above, to gust loads or gravity forces) requires attention. Only root constraints were studied here, but methods are already available for more general situations. ${ }^{12}$ Third, the linearization of the equations is carried out on the intrinsic variables, and it can in principle be independent of any linearization of the kinematic relations that link the velocities or internal strains with the actual displacement field in the beam elements. This could complicate the interpretation of linearized results given in velocities, but a linear velocity-displacement relation can always be assumed in such cases. As we have shown in this paper, results from the intrinsic formulation match those of displacement-based theories with a much simpler description. The modal solution in particular is expected to be a good basis for the design of flight control systems for vehicles with large wing displacements.

\section{References}

${ }^{1}$ Drela, M., "Integrated simulation model for preliminary aerodynamic, structural, and control-law design of aircraft," 40th AIAA/ASME/ASCE/AHS/ASC Structures, Structural Dynamics and Materials Conference, St. Louis, Missouri, USA. AIAA Paper 1999-1394, April 1999.

${ }^{2}$ Patil, M. J., Hodges, D., and Cesnik, C., "Nonlinear Aeroelastic Analysis of Complete Aircraft in Subsonic Flow," Journal of Aircraft, Vol. 37, No. 5, 2000, pp. 753-760.

${ }^{3}$ Shearer, C. and Cesnik, C., "Nonlinear Flight Dynamics of Very Flexible Aircraft," Journal of Aircraft, Vol. 44, No. 5, 2007, pp. 1528-1545.

${ }^{4}$ Stevens, B. L. and Lewis, F. L., Aircraft Control and Simulation, John Wiley \& Sons, Inc., New York, NY, USA, 1992.

${ }^{5}$ Hegemier, G. and Nair, S., "A Nonlinear Dynamical Theory for Heterogeneous, Anisotropic, Elastic Rods," AIAA Journal, Vol. 15, No. 1, 1977, pp. 8-15.

${ }^{6}$ Hodges, D., "Geometrically exact, intrinsic theory for dynamics of curved and twisted anisotropic beams," AIAA Journal, Vol. 41, No. 6, 2003, pp. 1131-1137.

${ }^{7}$ Love, A. E. H., A Treatise on the Mathematical Theory of Elasticity, Dover Publications Inc, New York, NY, USA, 4th ed., 1944, (First published in 1927 by Cambridge University Press).

${ }^{8}$ Chang, C.-S., Hodges, D. H., and Patil, M. J., "Flight dynamics of highly flexible aircraft," Journal of Aircraft, Vol. 45, No. 2, 2008, pp. 538-545.

${ }^{9}$ Palacios, R., "Nonlinear normal modes in an intrinsic theory of anisotropic beams," Journal of Sound and Vibration, Vol. 330, No. 8, 2011, pp. 1772-1792.

${ }^{10}$ Patil, M. J. and Hodges, D., "Flight Dynamics of Highly Flexible Flying Wings," Journal of Aircraft, Vol. 43, No. 6, 2006, pp. 1790-1798.

${ }^{11}$ Sotoudeh, Z., Hodges, D., and Chang, C.-S., "Validation Studies for Aeroelastic Trim and Stability Analysis of Highly Flexible Aircraft," Journal of Aircraft, Vol. 47, No. 4, 2010, pp. 1240-1247. 
${ }^{12}$ Sotoudeh, Z. and Hodges, D., "Nonlinear Aeroelastic Analysis of Joined-Wing Aircraft with Fully Intrinsic Equations," 50th AIAA/ASME/ASCE/AHS/ASC Structures, Structural Dynamics, and Materials Conference, Palm Springs, California, AIAA Paper 2009-2464, May 2009.

${ }^{13}$ Palacios, R. and Cesnik, C., "Cross-Sectional Analysis of Non-Homogeneous Anisotropic Active Slender Structures," AIAA Journal, Vol. 43, No. 12, 2005, pp. 2624-2638.

${ }^{14}$ Leishman, J., "Unsteady Lift of a Flapped Airfoil by Indicial Concepts," Journal of Aircraft, Vol. 31, No. 2, 1994, pp. 288-297.

${ }^{15}$ Shang, X. and Hodges, D., "Aeroelastic stability of composite hingeless rotors with advanced configurations," $37 t h$ AIAA/ASME/ASCE/AHS/ASC Structures, Structural Dynamics, and Materials Conference. Salt Lake City, Utah, USA, AIAA Paper 1996-1548, April 1996.

${ }^{16}$ Peters, D. A., "Two-dimensional Incompressible Unsteady Airfoil Theory - An Overview," Journal of Fluids and Structures, Vol. 24, No. 3, 2008, pp. 295-312.

${ }^{17}$ Pai, P. F., Highly Flexible Structures - Modeling, Computation, and Experimentation, AIAA Education Series, American Institute of Aeronautics and Astronautics, Reston, Virginia, USA, 2007.

${ }^{18}$ Palacios, R., Murua, J., and Cook, R., "Structural and Aerodynamic Models in the Nonlinear Flight Dynamics of Very Flexible Aircraft," AIAA Journal, Vol. 48, No. 11, November 2010, pp. 2648-2559.

${ }^{19}$ Goland, M., "The Flutter of a Cantilever Wing," Journal of Applied Mechanics, Vol. 12, No. 4, 1945, pp. $197-208$.

${ }^{20}$ Wang, Z., Chen, P., Liu, D., Mook, D., and Patil, M., "Time Domain Nonlinear Aeroelastic Analysis for HALE Wings," 47th AIAA/ASME/ASCE/AHS/ASC Structures, Structural Dynamics, and Materials Conference, Newport, Rhode Island, AIAA Paper 2006-1640, May 2006.

${ }^{21}$ Murua, J., Palacios, R., and Graham, J., "Modeling of Nonlinear Flexible Aircraft Dynamics Including Free-Wake Effects," AIAA Guidance, Navigation, and Control Conference 2 - 5 August 2010, Toronto, Ontario Canada, 2010.

${ }^{22}$ Murua, J., Hesse, H., Palacios, R., and Graham, J., "Stability and Open-Loop Dynamics of Very Flexible Aircraft Including Free-Wake Effects," 52nd AIAA/ASME/ASCE/AHS/ASC Structures, Structural Dynamics and Materials Conference, Denver, Colorado, USA, April 2011.

${ }^{23}$ Eastep, F. and Olsen, J., "Transonic Flutter Analysis of a Rectangular Wing with Conventional Airfoil Sections," AIAA Journal, Vol. 17, No. 10, 1984, pp. 1159-1164.

\section{Appendix}

Using the solution process of section III.A, closed-form expressions can be easily obtained for the LNMs in the intrinsic description of a constant section Euler-Bernoulli beam with sectional constants

$$
\begin{aligned}
\mathbf{m} & =\operatorname{diag}\left\{\rho A, \rho A, \rho A, \rho I_{1}, 0,0\right\}, \\
\mathbf{c}^{-1} & =\operatorname{diag}\left\{E A, 0,0, G J, E I_{2}, E I_{3}\right\} .
\end{aligned}
$$

They are shown next for the case of a cantilever beam on a moving base along the $y$ axis.

\section{Axial modes.}

The corresponding non-zero degrees of freedom in Eqs. (15) are then

$$
\left\{\begin{array}{l}
\Phi_{V_{1}} \\
\Phi_{F_{1}}
\end{array}\right\}=\exp \left(\left[\begin{array}{cc}
0 & -\frac{\omega}{E A} \\
\rho A \omega & 0
\end{array}\right] x\right)\left\{\begin{array}{l}
\Phi_{V_{1}}(0) \\
\Phi_{F_{1}}(0)
\end{array}\right\} .
$$

This equation is solved with clamped-free conditions, that is, $\Phi_{V_{1}}(0)=0$ and $\Phi_{F_{1}}(L)=0$. The eigenvalues of this problem are $\omega_{j}=\sqrt{\frac{E}{\rho}} \lambda_{j}$, with $\lambda_{j}=\frac{2 j-1}{2} \frac{\pi}{L}$ and $j=0,1,2, \ldots, \infty$. The corresponding eigenvectors, after normalization, are

$$
\begin{aligned}
\Phi_{V_{1} j} & =\sqrt{\frac{2}{\rho A L}} \sin \left(\lambda_{j} x\right), \\
\Phi_{F_{1} j} & =-\sqrt{\frac{2 E A}{L}} \cos \left(\lambda_{j} x\right) .
\end{aligned}
$$

\section{Torsional modes.}

Eqs. (15) reduce in this case to

$$
\left\{\begin{array}{l}
\Phi_{\Omega_{1}} \\
\Phi_{M_{1}}
\end{array}\right\}=\exp \left(\left[\begin{array}{cc}
0 & -\frac{\omega}{G J} \\
\rho I_{1} \omega & 0
\end{array}\right] s\right)\left\{\begin{array}{l}
\Phi_{\Omega_{1}}(0) \\
\Phi_{M_{1}}(0)
\end{array}\right\},
$$


with $\Phi_{\Omega_{1}}(0)=0$ and $\Phi_{M_{1}}(L)=0$. These equations are identical to those of the axial modes but with different coefficients. Frequencies and modes can be obtained directly from the previous results.

\section{Bending modes in the $(x, y)$ plane (slide-free)}

In this case, it is

$$
\left\{\begin{array}{l}
\Phi_{V_{2}} \\
\Phi_{\Omega_{3}} \\
\Phi_{F_{2}} \\
\Phi_{M_{3}}
\end{array}\right\}=\exp \left(\left[\begin{array}{cccc}
0 & 1 & 0 & 0 \\
0 & 0 & 0 & -\frac{\omega}{E I_{3}} \\
\rho A \omega & 0 & 0 & 0 \\
0 & 0 & -1 & 0
\end{array}\right] x\right)\left\{\begin{array}{c}
\bar{\Phi}_{V_{2}} \\
\bar{\Phi}_{\Omega_{3}} \\
\bar{\Phi}_{F_{2}} \\
\bar{\Phi}_{M_{3}}
\end{array}\right\}
$$

The slide-free conditions are $\Phi_{F_{2}}(0)=0, \Phi_{\Omega_{3}}(0)=0, \Phi_{F_{2}}(L)=0$ and $\left.\Phi_{M_{3}}(L)=0\right)$. Defining $\omega_{j}=$ $\left(\lambda_{j}\right)^{2} \sqrt{\frac{E I_{3}}{\rho A}}$, the eigenvalues for this problem are the roots of the characteristic equation,

$$
\cos \left(\lambda_{j} L\right) \sinh \left(\lambda_{j} L\right)+\sin \left(\lambda_{j} L\right) \cosh \left(\lambda_{j} L\right)=0,
$$

and the corresponding mode shapes are

$$
\begin{aligned}
\Phi_{V_{2} j} & =\frac{k_{j}}{\sqrt{\rho A L}}\left[\cos \left(\lambda_{j} x\right)+\cosh \left(\lambda_{j} x\right)-\Lambda_{j}\left(\cos \left(\lambda_{j} x\right)-\cosh \left(\lambda_{j} x\right)\right)\right], \\
\Phi_{\Omega_{3} j} & =\frac{k_{j} \lambda_{j}}{\sqrt{\rho A L}}\left[-\sin \left(\lambda_{j} x\right)+\sinh \left(\lambda_{j} x\right)+\Lambda_{j}\left(\sin \left(\lambda_{j} x\right)+\sinh \left(\lambda_{j} x\right)\right)\right], \\
\Phi_{F_{2} j} & =k_{j} \lambda_{j} \sqrt{\frac{E I_{3}}{L}}\left[\sin \left(\lambda_{j} x\right)+\sinh \left(\lambda_{j} x\right)-\Lambda_{j}\left(\sin \left(\lambda_{j} x\right)-\sinh \left(\lambda_{j} x\right)\right)\right], \\
\Phi_{M_{3} j} & =k_{j} \sqrt{\frac{E I_{3}}{L}}\left[\cos \left(\lambda_{j} x\right)-\cosh \left(\lambda_{j} x\right)-\Lambda_{j}\left(\cos \left(\lambda_{j} x\right)+\cosh \left(\lambda_{j} x\right)\right)\right],
\end{aligned}
$$

with $j=1,2, \ldots, \infty$ corresponding to the positive roots of Eq. (33), and with

$$
\begin{aligned}
\Lambda_{j} & =\frac{\cos \left(\lambda_{j} L\right)-\cosh \left(\lambda_{j} L\right)}{\cos \left(\lambda_{j} L\right)+\cosh \left(\lambda_{j} L\right)}, \\
k_{j}^{2} & =\frac{\left(\cos \left(\lambda_{j} L\right)+\cosh \left(\lambda_{j} L\right)\right)^{2}}{2\left(\cos ^{2}\left(\lambda_{j} L\right)+\cosh ^{2}\left(\lambda_{j} L\right)\right)}
\end{aligned}
$$

This problem also has a rigid-body mode $\left(\omega_{0}=0\right)$ as a solution. The only non-zero component of the normalized mode shape is

$$
\Phi_{V_{2} 0}(s)=\frac{1}{\sqrt{\rho A L}} .
$$

\section{Bending modes in the (x,z) plane (clamped-free)}

Finally, bending modes for clamped-free case are obtained in a similar way as above. They are included here for completeness. The natural frequecies are $\omega_{j}=\left(\lambda_{j}\right)^{2} \sqrt{\frac{E I_{2}}{\rho A}}$, where $\lambda_{j}$ are the solutions to

$$
\cos \left(\lambda_{j} L\right) \cosh \left(\lambda_{j} L\right)+1=0 .
$$

The corresponding eigenvectors are

$$
\begin{aligned}
\Phi_{V_{3} j} & =\frac{1}{\sqrt{\rho A L}}\left[\cos \left(\lambda_{j} x\right)-\cosh \left(\lambda_{j} x\right)-\Lambda_{j}\left(\sin \left(\lambda_{j} x\right)-\sinh \left(\lambda_{j} x\right)\right)\right], \\
\Phi_{\Omega_{2} j} & =\frac{\lambda_{j}}{\sqrt{\rho A L}}\left[\sin \left(\lambda_{j} x\right)+\sinh \left(\lambda_{j} x\right)+\Lambda_{j}\left(\cos \left(\lambda_{j} x\right)-\cosh \left(\lambda_{j} x\right)\right)\right], \\
\Phi_{F_{3} j} & =\lambda_{j} \sqrt{\frac{E I_{2}}{L}}\left[\sin \left(\lambda_{j} x\right)-\sinh \left(\lambda_{j} x\right)-\Lambda_{j}\left(\cos \left(\lambda_{j} x\right)+\cosh \left(\lambda_{j} x\right)\right)\right], \\
\Phi_{M_{2} j} & =\sqrt{\frac{E I_{2}}{L}}\left[-\cos \left(\lambda_{j} x\right)-\cosh \left(\lambda_{j} x\right)+\Lambda_{j}\left(\sin \left(\lambda_{j} x\right)+\sinh \left(\lambda_{j} x\right)\right)\right],
\end{aligned}
$$


with

$$
\Lambda_{j}=\frac{\cos \left(\lambda_{j} L\right)+\cosh \left(\lambda_{j} L\right)}{\sin \left(\lambda_{j} L\right)+\sinh \left(\lambda_{j} L\right)}
$$

\section{Acknowledgments}

Many thanks to Dr Paul Goulart, from the Eidgenössische Technische Hochschule (ETH) Zürich, for his careful review of the structural model and suggestions for notation. He also identified the symmetry conditions satisfied by the coefficients of the nonlinear modal equations used in the paper. 